Bull. Korean Math. Soc. 52 (2015), No. 5, pp. 1661-1668

http://dx.doi.org/10.4134/BKMS.2015.52.5.1661

\title{
RULED SURFACES AND GAUSS MAP
}

\author{
DonG-SoO KIM
}

\begin{abstract}
We study the Gauss map $G$ of ruled surfaces in the 3-dimensional Euclidean space $\mathbb{E}^{3}$ with respect to the so called Cheng-Yau operator $\square$ acting on the functions defined on the surfaces. As a result, we establish the classification theorem that the only ruled surfaces with Gauss map $G$ satisfying $\square G=A G$ for some $3 \times 3$ matrix $A$ are the flat ones. Furthermore, we show that the only ruled surfaces with Gauss map $G$ satisfying $\square G=A G$ for some nonzero $3 \times 3$ matrix $A$ are the cylindrical surfaces.
\end{abstract}

\section{Introduction}

The theory of Gauss map of surfaces in the $n$-dimensional Euclidean space $\mathbb{E}^{n}$ or in the $n$-dimensional Lorentz-Minkowski space $\mathbb{L}^{n}$ is always one of interesting topics and it has been investigated from various viewpoints by a lot of differential geometers $([2,4,7,9,10,11,14,15,16,17,18,21,24,25])$.

We denote by $M$ a surface of the Euclidean 3 -space $\mathbb{E}^{3}$. The map $G: M \rightarrow$ $S^{2} \subset \mathbb{E}^{3}$ which sends each point $p$ of $M$ to the unit normal vector $G(p)$ to $M$ at $p$ is called the Gauss map of the surface $M$, where $S^{2}$ is the unit sphere in $\mathbb{E}^{3}$ centered at the origin. It is well known that $M$ has constant mean curvature if and only if $\Delta G=\|d G\|^{2} G$, where $\Delta$ is the Laplace operator on M with respect to the induced metric on the surface $\mathrm{M}$ from $\mathbb{E}^{3}$ ([26]). As a special case, one can consider surfaces whose Gauss map is an eigenfunction of a Laplacian, that is, $\Delta G=\lambda G$ for some constant $\lambda \in R$. Generalizing this equation, F. Dillen, J. Pas and L. Verstraelen ([12]) studied surfaces of revolution in the Euclidean 3 -space $\mathbb{E}^{3}$ whose Gauss map G satisfies the condition

$$
\Delta G=A G, \quad A \in R^{3 \times 3} .
$$

As a result, they proved ([12]):

Proposition 1.1. Among the surfaces of revolution in $\mathbb{E}^{3}$, the only ones whose Gauss map satisfies (1.1) are the planes, the spheres and the circular cylinders.

Received November 4, 2014.

2010 Mathematics Subject Classification. 53A05, 53B25.

Key words and phrases. Gauss map, Cheng-Yau operator, ruled surface.

This study was financially supported by Chonnam National University, 2014. 

$([3])$.

C. Baikoussis and D. E. Blair also studied ruled surfaces in $\mathbb{E}^{3}$ and proved

Proposition 1.2. Among the ruled surfaces in $\mathbb{E}^{3}$, the only ones whose Gauss map satisfies (1.1) are the planes and the circular cylinders.

In [20], generalized slant cylindrical surfaces (GSCS's) were introduced, which are natural extended notion of surfaces of revolution. Surfaces of revolution, cylindrical surfaces and tubes along a plane curve are special cases of GSCS's. Generalizing Proposition 1.1, the author and B. Song proved that among the GSCS's in $\mathbb{E}^{3}$, the only ones whose Gauss map satisfies (1.1) are the planes, the spheres and the circular cylinders $([22])$.

The so-called Cheng-Yau operator $\square$ (or, $L_{1}$ ) is a natural extension of the Laplace operator $\Delta$ (cf. [1], [8]). Hence, following the condition (1.1), an interesting geometric question is raised as follows.

Question 1.3. Among ruled surfaces in the Euclidean 3-space $\mathbb{E}^{3}$, which one satisfy the following condition?

$$
\square G=A G, \quad A \in R^{3 \times 3} .
$$

In this paper, we give a complete answer to the above question as follows.

Theorem 1.4. Let $M$ denote a ruled surface in the Euclidean 3-space $\mathbb{E}^{3}$. Then the Gauss map of $M$ satisfies (1.2) for a matrix $A$ if and only if it is a developable surface.

Theorem 1.5. Suppose that $M$ denotes a ruled surface in the Euclidean 3space $\mathbb{E}^{3}$. Then the Gauss map of $M$ satisfies (1.2) for some nonzero matrix $A$ if and only if it is a cylindrical surface.

For surfaces of revolution in the Euclidean 3 -space $\mathbb{E}^{3}$, recently the author et al. established the following ([19]):

Proposition 1.6. Let $M$ be a surface of revolution. Then the Gauss map $G$ of $M$ satisfies $\square G=A G$ for some $3 \times 3$ matrix $A$ if and only if $M$ is an open part of the following surfaces:

1) a plane,

2) a right circular cone,

3) a circular cylinder,

4) a sphere.

Throughout this paper, we assume that all objects are smooth and connected, unless otherwise mentioned.

\section{Cheng-Yau operator and lemmas}

Suppose that $M$ denotes an oriented surface in the Euclidean 3 -space $\mathbb{E}^{3}$ with Gauss map $G$. We denote by $S$ the shape operator of $M$ with respect to the Gauss map $G$. For each $k=0,1$, we put $P_{0}=I, P_{1}=\operatorname{tr}(S) I-S$, where $I$ is the 
identity operator acting on the tangent bundle of $M$. Let us define an operator $L_{k}: C^{\infty}(M) \rightarrow C^{\infty}(M)$ by $L_{k}(f)=\operatorname{tr}\left(P_{k} \circ \nabla^{2} f\right)$, where $\nabla^{2} f: \chi(M) \rightarrow \chi(M)$ denotes the self-adjoint linear operator metrically equivalent to the hessian of $f$. Then, up to signature, $L_{k}$ is the linearized operator of the first variation of the $(k+1)$-th mean curvature arising from normal variations of the surface. When $k=0$, the operator $L_{0}$ is nothing but the Laplace operator acting on $M$, i.e., $L_{0}=\Delta$. If $k=1, L_{1}=\square$ is called the Cheng-Yau operator introduced in [8].

In order to prove our theorems in Sections 3 and 4, we need the following useful lemma $([1,23])$.

Lemma 2.1. Let $M$ denote an oriented surface in $\mathbb{E}^{3}$ with Gaussian curvature $K$ and mean curvature $H$. Then, the Gauss map $G$ of $M$ satisfies

$$
-\square G=\nabla K+2 H K G,
$$

where $\nabla K$ denotes the gradient of $K$.

Now, using Lemma 2.1 we give some examples of surfaces with Gauss map satisfying (1.2).

Example 2.2. (1) Flat surfaces: In this case, we have $\square G=0$, and hence flat surfaces satisfy $\square G=A G$ for some $3 \times 3$ matrix $A$. Note that the matrix $A$ must be singular.

(2) Spheres: $(x-a)^{2}+(y-b)^{2}+(z-c)^{2}=r^{2}$. In this case, we have $G=\frac{1}{r}(x-a, y-b, z-c)$ so the sphere satisfies $\square G=A G$ with $A=\frac{2}{r^{3}} I$, where $I$ denotes the identity matrix.

(3) Cylindrical surfaces: For a unit speed plane curve $\alpha(s)=(x(s), y(s), 0)$, we consider the cylindrical surface $M$ defined by $X(s, t)=(x(s), y(s), t)$. In this case, we have $G=\left(-y^{\prime}(s), x^{\prime}(s), 0\right)$ so the surface $M$ satisfies $\square G=A G$ for some nonzero matrix $A$ of the following form:

$$
A=\left(\begin{array}{lll}
0 & 0 & * \\
0 & 0 & * \\
0 & 0 & *
\end{array}\right) .
$$

\section{Proof of Theorem 1.4}

We suppose that $M$ is a ruled surface in the Euclidean 3-space $\mathbb{E}^{3}$. The surface $M$ can be expressed in terms of a directrix curve $\alpha(s)$ and a unit vector field $\beta(s)$ pointing along the rulings as

$$
X(s, t)=\alpha(s)+t \beta(s) .
$$

When $M$ is cylindrical, that is, the direction vector field $\beta$ is constant, the Gaussian curvature $K$ vanishes. Hence $M$ satisfies (1.2) trivially.

Henceforth, we consider only the ruled surface $M$ which is not cylindrical, that is, $\beta^{\prime}(s)$ never vanish. Thus, we can choose the parameter $s$ so that $\beta(s)$ 
is of unit speed. For such parameter $s$ of $\beta$, we also choose an orthogonal trajectory $\alpha$ to the rulings. Hence, we have

$$
\left\langle\alpha^{\prime}, \beta\right\rangle=0,\langle\beta, \beta\rangle=\left\langle\beta^{\prime}, \beta^{\prime}\right\rangle=1 \text {. }
$$

We put $u(s)=\left\langle\alpha^{\prime}(s), \beta^{\prime}(s)\right\rangle$ and $v(s)=\left\langle\alpha^{\prime}(s), \alpha^{\prime}(s)\right\rangle$. Then the Gauss map $G$ of $M$ is given by

$$
G=Q^{-1 / 2}\left\{\left(\alpha^{\prime} \times \beta\right)+t\left(\beta^{\prime} \times \beta\right)\right\},
$$

where $Q=\left|\alpha^{\prime}+t \beta^{\prime}\right|^{2}=t^{2}+2 u t+v$. It is straightforward to show that the Gaussian curvature $K$ is given by $([13])$

$$
K=Q^{-2} w,
$$

where we use $w=u^{2}-v$.

For the mean curvature $H$ of $M$, first we note that the Laplacian $\Delta$ of $M$ can be expressed as

$$
-\Delta=\frac{\partial^{2}}{\partial t^{2}}+\frac{1}{Q} \frac{\partial^{2}}{\partial s^{2}}-\frac{1}{2 Q^{2}} \frac{\partial Q}{\partial s} \frac{\partial}{\partial s}+\frac{1}{2 Q} \frac{\partial Q}{\partial t} \frac{\partial}{\partial t} .
$$

Using the well-known Laplace-Beltrami equation $([5,6])$

$$
-\Delta X=2 H G,
$$

it follows from (3.1) and (3.5) that

$$
2 H=Q^{-3 / 2} P(t),
$$

where $P(t)=a_{0} t^{2}+a_{1} t+a_{2}$ with coefficients given by

(3.8) $a_{0}=\left\langle\beta^{\prime \prime}, \beta^{\prime} \times \beta\right\rangle, a_{1}=\left\langle\alpha^{\prime \prime}, \beta^{\prime} \times \beta\right\rangle+\left\langle\beta^{\prime \prime}, \alpha^{\prime} \times \beta\right\rangle, a_{2}=\left\langle\alpha^{\prime \prime}, \alpha^{\prime} \times \beta\right\rangle$.

In order to get the expression of $\nabla K$, we use the orthonormal frame $\left\{e_{1}, e_{2}\right\}$ of $M$ given by

$$
e_{1}=Q^{-1 / 2} X_{s}, e_{2}=X_{t},
$$

where $X_{s}$ means the derivative of $X$ with respect to $s$, etc.. Then we get

$$
\nabla K=e_{1}(K) e_{1}+e_{2}(K) e_{2}=Q^{-4} R(t),
$$

where $R(t)$ is a vector-valued polynomial in $t$ of $\operatorname{deg}(R) \leq 3$ which is given by

$$
R(t)=\left\{Q w^{\prime}-2 w\left(2 u^{\prime} t+v^{\prime}\right)\right\}\left(\alpha^{\prime}+t \beta^{\prime}\right)-4 Q(t+u) w \beta .
$$

Together with (3.3), (3.4), (3.7) and (3.10), Lemma 2.1 shows that

$$
\square G=-Q^{-4}\left[R(t)+w P(t)\left\{\left(\alpha^{\prime} \times \beta\right)+t\left(\beta^{\prime} \times \beta\right)\right\}\right] .
$$

Now, suppose that the Gauss map $G$ of $M$ satisfies $\square G=A G$ for some $3 \times 3$ matrix $A$. Then, from (3.3) and (3.12) we have

(3.13) $R(t)+w P(t)\left\{\left(\alpha^{\prime} \times \beta\right)+t\left(\beta^{\prime} \times \beta\right)\right\}=-Q^{7 / 2}\left\{A\left(\alpha^{\prime} \times \beta\right)+t A\left(\beta^{\prime} \times \beta\right)\right\}$. By considering the degrees in $t$ of both sides of (3.13), we can conclude

$$
A\left(\alpha^{\prime} \times \beta\right)=A\left(\beta^{\prime} \times \beta\right)=0,
$$


and hence we obtain

$$
R(t)+w P(t)\left\{\left(\alpha^{\prime} \times \beta\right)+t\left(\beta^{\prime} \times \beta\right)\right\}=0 .
$$

We consider the coefficient of $t^{3}$ in the equation (3.15). Then from (3.8) and (3.11) we have

$$
w^{\prime} \beta^{\prime}-4 w \beta+a_{0} w\left(\beta^{\prime} \times \beta\right)=0 .
$$

Since $\beta^{\prime}, \beta$ and $\beta^{\prime} \times \beta$ are linearly independent, it follows from (3.16) that $w$ vanishes identically. Therefore (3.4) implies that $M$ is flat, and hence it is developable.

The converse follows from Example 2.2. This completes the proof of Theorem 1.4 .

\section{Proof of Theorem 1.5}

In this section, we prove Theorem 1.5.

First of all, we need the following lemma.

Lemma 4.1. Let $M$ be a non-cylindrical ruled surface in the Euclidean 3-space $\mathbb{E}^{3}$ which is given by

$$
X(s, t)=\alpha(s)+t \beta(s),
$$

where $\alpha$ and $\beta$ satisfy (3.2). Suppose that the Gauss map $G$ of $M$ satisfies (1.2) for some nonzero $3 \times 3$ matrix $A$. Then $M$ is nothing but an open part of a plane.

Proof. We use the same notations as in Section 3. From the arguments there, we get $w=u^{2}-v=0$, and hence $Q=(t+u)^{2}$. Since $\left\{\beta^{\prime}, \beta, \beta^{\prime} \times \beta\right\}$ is orthonormal, from (3.2) we have

$$
\alpha^{\prime}=u \beta^{\prime}+\left\langle\alpha^{\prime}, \beta^{\prime} \times \beta\right\rangle \beta^{\prime} \times \beta .
$$

This shows that

$$
v=\left\langle\alpha^{\prime}, \alpha^{\prime}\right\rangle=u^{2}+\left\langle\alpha^{\prime}, \beta^{\prime} \times \beta\right\rangle^{2} .
$$

Hence we get

$$
\alpha^{\prime}=u \beta^{\prime} .
$$

Together with (3.3), (4.4) shows that

$$
G=\beta^{\prime} \times \beta .
$$

If we denote by $V$ the kernel space of the matrix $A$, then (3.14) implies that $\beta^{\prime} \times \beta$ lies in the space $V$. By assumption, there exists a unit vector $a$ (for simplicity, say $a=(0,0,1)$ ) which is orthogonal to $V$. We put $\beta(s)=$ $(x(s), y(s), z(s))$. Then we have

$$
\left\langle a, \beta^{\prime} \times \beta\right\rangle=x^{\prime} y-x y^{\prime}=0 .
$$


We denote by $J=\{s \in I \mid(x(s), y(s)) \neq 0\}$, where $I$ is the domain of $s$. Then, on $J$ (4.6) shows that for some function $f=f(s)$

$$
\left(x^{\prime}, y^{\prime}\right)=f(x, y) \text {. }
$$

This, together with (3.2), leads to the following:

$$
f^{2}=\frac{1-\left(z^{\prime}\right)^{2}}{1-z^{2}}
$$

Moreover, it follows from (3.2), (4.7) and $\left\langle\beta^{\prime}, \beta\right\rangle=0$ that

$$
f=\frac{-z z^{\prime}}{1-z^{2}} .
$$

We eliminate the function $f$ from (4.8) and (4.9). Then we get

$$
\left(z^{\prime}\right)^{2}=1-z^{2} \text {. }
$$

Integrating (4.10), we obtain

$$
z(s)= \pm \sin (s+c),
$$

where $c$ is a constant. This shows that

$$
x^{2}+y^{2}=\cos ^{2}(s+c),
$$

and hence we have for some function $\theta=\theta(s)$

$$
\beta(s)=(\cos \theta(s) \cos (s+c), \sin \theta(s) \cos (s+c), \pm \sin (s+c)) .
$$

Differentiating (4.13) and using $\left\langle\beta^{\prime}, \beta^{\prime}\right\rangle=1$, we see that $\theta^{\prime}(s)=0$. Thus we have

$$
\beta(s)=(\cos d \cos (s+c), \sin d \cos (s+c), \pm \sin (s+c)),
$$

where $d$ is a constant.

It follows from (4.14) that on the subinterval $J$ of $I, \beta^{\prime} \times \beta$ is a constant vector. Since $\beta^{\prime}(s) \neq 0$ for $s \in I$, we see that the complement $J^{c}$ of $J$ has no interior points. This shows that $\beta^{\prime} \times \beta$ is constant on the whole interval $I$ of s. Therefore, (4.5) completes the proof of Lemma 4.1 .

Finally, we prove Theorem 1.5.

Let us denote by $M$ a ruled surface in the Euclidean 3 -space $\mathbb{E}^{3}$. Suppose that the Gauss map of $M$ satisfies (1.2) for some nonzero matrix $A$. Then Lemma 4.1 shows that $M$ is either a cylindrical surface or an open part of a plane. Since a plane can be reparametrized so that the directrix curve $\alpha$ is a straight line and hence the direction vector field $\beta$ is constant, $M$ is cylindrical. This completes the only if part of Theorem 1.5.

Conversely, the if part of Theorem 1.5 follows from Example 2.2. This completes the proof of Theorem 1.5. 


\section{References}

[1] L. J. Alias and N. Gurbuz, An extension of Takahashi theorem for the linearized operators of the higher order mean curvatures, Geom. Dedicata 121 (2006), 113-127.

[2] C. Baikoussis, Ruled submanifolds with finite type Gauss map, J. Geom. 49 (1994), no. $1-2,42-45$.

[3] C. Baikoussis and D. E. Blair, On the Gauss map of ruled surfaces, Glasgow Math. J. 34 (1992), no. 3, 355-359.

[4] C. Baikoussis and L. Verstraelen, On the Gauss map of helicoidal surfaces, Rend. Sem. Mat. Messina Ser. II 2(16) (1993), 31-42.

[5] B.-Y. Chen, Total Mean Curvature and Submanifolds of Finite Type, World Scientific Publ., New Jersey, 1984.

[6] Finite Type Submanifolds and Generalizations, University of Rome, 1985.

[7] B.-Y. Chen and P. Piccinni, Submanifolds with finite type Gauss map, Bull. Austral. Math. Soc. 35 (1987), no. 2, 161-186.

[8] S. Y. Cheng and S. T. Yau, Hypersurfaces with constant scalar curvature, Math. Ann. 225 (1977), no. 3, 195-204.

[9] M. Choi, D.-S. Kim, Y. H. Kim, and D. W. Yoon, Circular cone and its Gauss map, Colloq. Math. 129 (2012), no. 2, 203-210.

[10] S. M. Choi, On the Gauss map of surfaces of revolution in a 3-dimensional Minkowski space, Tsukuba J. Math. 19 (1995), no. 2, 351-367.

[11] - On the Gauss map of ruled surfaces in a 3-dimensional Minkowski space, Tsukuba J. Math. 19 (1995), no. 2, 285-304.

[12] F. Dillen, J. Pas, and L. Verstraelen, On the Gauss map of surfaces of revolution, Bull. Inst. Math. Acad. Sinica 18 (1990), no. 3, 239-246.

[13] M. P. do Carmo, Differential Geometry of Curves and Surfaces, Translated from the Portuguese, Prentice-Hall, Inc., Englewood Cliffs, N.J., 1976.

[14] U. Dursun, Hypersurfaces with pointwise 1-type Gauss map, Taiwanese J. Math. 11 (2007), no. 5, 1407-1416.

[15] Flat surfaces in the Euclidean space $E^{3}$ with pointwise 1-type Gauss map, Bull. Malays. Math. Sci. Soc. (2) 33 (2010), no. 3, 469-478.

[16] U.-H. Ki, D.-S. Kim, Y. H. Kim, and Y.-M. Roh, Surfaces of revolution with pointwise 1-type Gauss map in Minkowski 3-space, Taiwanese J. Math. 13 (2009), no. 1, 317-338.

[17] D.-S. Kim, On the Gauss map of quadric hypersurfaces, J. Korean Math. Soc. 31 (1994), no. 3, 429-437.

[18] On the Gauss map of hypersurfaces in the space form, J. Korean Math. Soc. 32 (1995), no. 3, 509-518.

[19] D.-S. Kim, J. R. Kim, and Y. H. Kim, Cheng-Yau operator and Gauss map of surfaces of revolution, Bull. Malays. Math. Sci. Soc., To appear. arXiv:1411.2291

[20] D.-S. Kim and Y. H. Kim, Surfaces with planar lines of curvature, Honam Math. J. 32 (2010), no. 4, 777-790.

[21] D.-S. Kim, Y. H. Kim, and D. W. Yoon, Extended B-scrolls and their Gauss maps, Indian J. Pure Appl. Math. 33 (2002), no. 7, 1031-1040.

[22] D.-S. Kim and B. Song, On the Gauss map of generalized slant cylindrical surfaces, J. Korea Soc. Math. Educ. Ser. B Pure Appl. Math. 20 (2013), no. 3, 149-158.

[23] Y. H. Kim and N. C. Turgay, Surfaces in $E^{3}$ with $L_{1}$-pointwise 1-type Gauss map, Bull. Korean Math. Soc. 50 (2013), no. 3, 935-949.

[24] Classifications of helicoidal surfaces with $L_{1}$-pointwise 1-type Gauss map, Bull. Korean Math. Soc. 50 (2013), no. 4, 1345-1356.

[25] Y. H. Kim and D. W. Yoon, On the Gauss map of ruled surfaces in Minkowski space, Rocky Mountain J. Math. 35 (2005), no. 5, 1555-1581.

[26] E. A. Ruh and J. Vilms, The tension field of the Gauss map, Trans. Amer. Math. Soc. 149 (1970), 569-573. 
Department of Mathematics

Chonnam National University

Gwanguu 61186, Korea

E-mail address: dosokim@chonnam.ac.kr 